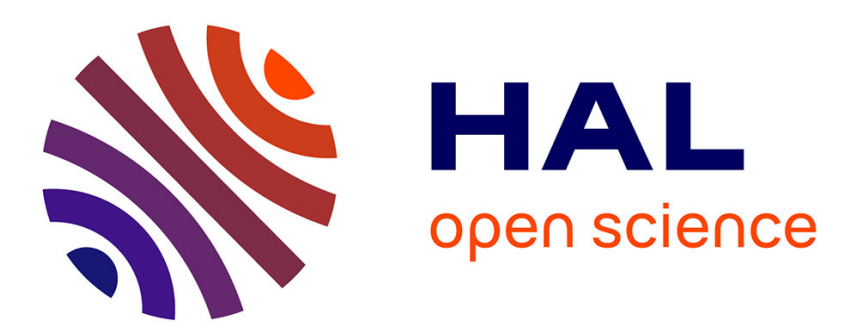

\title{
Ranking-based semantics for argumentation frameworks
}

Leila Amgoud, Jonathan Ben-Naim

\section{To cite this version:}

Leila Amgoud, Jonathan Ben-Naim. Ranking-based semantics for argumentation frameworks. 7th International Conference on Scalable Uncertainty Management (SUM 2013), Sep 2013, Washington, DC, United States. pp.134-147. hal-01239719

\section{HAL Id: hal-01239719 https://hal.science/hal-01239719}

Submitted on 9 Dec 2015

HAL is a multi-disciplinary open access archive for the deposit and dissemination of scientific research documents, whether they are published or not. The documents may come from teaching and research institutions in France or abroad, or from public or private research centers.
L'archive ouverte pluridisciplinaire HAL, est destinée au dépôt et à la diffusion de documents scientifiques de niveau recherche, publiés ou non, émanant des établissements d'enseignement et de recherche français ou étrangers, des laboratoires publics ou privés. 


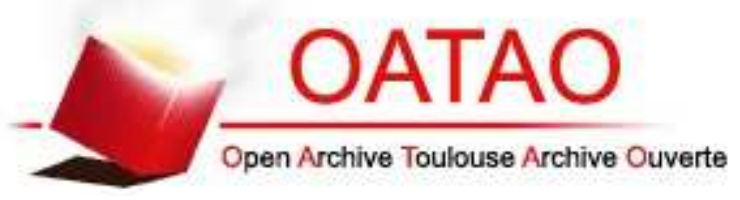

\section{Open Archive TOULOUSE Archive Ouverte (OATAO)}

OATAO is an open access repository that collects the work of Toulouse researchers and makes it freely available over the web where possible.

This is an author-deposited version published in : http://oatao.univ-toulouse.fr/ Eprints ID : 12512

The contribution was presented at SUM 2013 :

http://informatique.umons.ac.be/sum2013/

Official URL: http://dx.doi.org/10.1007/978-3-642-40381-1_11

To cite this version : Amgoud, Leila and Ben-Naim, Jonathan Ranking-based semantics for argumentation frameworks. (2013) In: 7th International Conference on Scalable Uncertainty Management (SUM 2013), 16 September 2013 - 18 September 2013 (Washington, DC, United States).

Any correspondance concerning this service should be sent to the repository administrator: staff-oatao@listes-diff.inp-toulouse.fr 


\title{
Ranking-based Semantics for Argumentation Frameworks
}

\author{
Leila Amgoud and Jonathan Ben-Naim
}

IRIT $-\mathrm{CNRS}^{\star \star}$

\begin{abstract}
An argumentation system consists of a set of interacting arguments and a semantics for evaluating them. This paper proposes a new family of semantics which rank-orders arguments from the most acceptable to the weakest one(s). The new semantics enjoy two other main features: i) an attack weakens its target but does not kill it, ii) the number of attackers has a great impact on the acceptability of an argument. We start by proposing a set of rational postulates that such semantics could satisfy, then construct various semantics that enjoy them.
\end{abstract}

\section{Introduction}

Argumentation is a reasoning model based on the construction and evaluation of interacting arguments. The most popular semantics were proposed by Dung in his seminal paper [6]. Those semantics as well as their refinements (e.g. in $[3,5])$ partition the powerset of the set of arguments into two classes: extensions and non-extensions. Every extension represents a coherent point of view. An absolute status is assigned to each argument: accepted (if it belongs to every extension), rejected (if it does not belong to any extension), and undecided if it is in some extensions and not in others. Those semantics are based in particular on the following considerations:

- Killing: The impact of an attack from an argument $b$ to an argument $a$ is drastic, that is, if $b$ belongs to an extension, then $a$ is automatically excluded from that extension (i.e., $a$ is killed).

- Existence: One successful attack against an argument $a$ has the same effect on $a$ as any number of successful attacks. Indeed, one such attack is sufficient to kill $a$, several attacks cannot kill $a$ to a greater extent.

- Absoluteness: The three possible status of the arguments are absolute, that is, they make sense even without comparing them with each other.

- Flatness: All the accepted arguments have the same level of acceptability.

These four considerations seem rational in applications like paraconsistent reasoning. For example, the killing consideration makes sense in this application, because arguments are formulas and attacks correspond to contradictions, and it is natural to consider that one contradiction is lethal.

However, in other applications, e.g. decision-making, some of these considerations are debatable. First, the killing principle is problematic in decision-making, because an attack does not necessarily kill its target, but just weakens it. Suppose for instance that the two following arguments $a$ and $b$ are exchanged by two doctors:

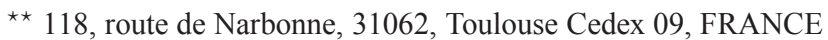


$a$ : The patient should have a surgery since he has cancer.

$b$ : The statistics show that the probability that a surgery will improve the state of the patient is low.

In this case, the attack from $b$ only weakens $a$, it does not kill $a$. The doctor may still choose to do the surgery since it gives (a small) chance for the patient to survive.

Next, the existence consideration is also debatable. Suppose a seller provides the following argument $a$ in favor of a given car:

$a$ : This car is certainly powerful since it is made by Peugeot.

$b_{1}$ : The engines of Peugeot cars break down before $300000 \mathrm{~km}$.

$b_{2}$ : The airbags of Peugeot cars are not reliable.

$b_{3}$ : The spare part is very expensive.

If the buyer receives the argument $b_{1}$ against Peugeot (thus against $a$ ), then he accepts less $a$. The situation becomes worse if he receives $b_{2}$ and $b_{3}$. Indeed, the more arguments he receives against $a$, the less his confidence in $a$.

The flatness consideration is also debatable in decision-making. Suppose for example that $a$ is not attacked, $b$ is attacked only by $a$, and $c$ is attacked only by $b$. Then, $a$ and $c$ are both accepted and have the same level of acceptability. But, in applications like decision-making, it is reasonable to consider that an attack from a non-attacked node (or any number of non-attacked nodes) does not kill the destination node. So, $b$ is only weakened, which means that its attack against $c$ should have some effect, that is, the level of acceptability of $c$ should be lower than that of $a$.

To sum up, existing semantics may be well-suited for reasoning but not for applications like decision-making. In the present paper, we propose a new family of semantics that are based on the following graded considerations:

- Weakening: Arguments cannot be killed (however, they can be weakened to an extreme extent). As a consequence, an attack from an argument $b$ to an argument $a$ always decreases the degree of acceptability of $a$ (possibly only by an infinitesimal amount). The greater the acceptability of $b$, the greater the decrease in the acceptability of $a$.

- Counting: The more numerous the attacks against $a$, the greater the decrease in the acceptability of $a$.

- Relativity: The degrees of acceptability of the arguments are relative, that is, they do not make sense when they are not compared with each other.

- Graduality: There is an arbitrarily large number of degrees of acceptability.

In our approach, a semantics is a function that transforms any argumentation graph into a ranking on its set of arguments: from the most accepted to the weakest one(s). Our first step consists in proposing formal postulates, each of which is an intuitive and desirable property that a semantics may enjoy. Our postulates are based on the four informal graded considerations described earlier: weakening, counting, relativity, and graduality. Such an axiomatic approach allows a better understanding of semantics and a more precise comparison between different proposals. We investigate dependencies and compatibilities between postulates. In a second step, we construct two rankingbased semantics satisfying certain postulates. 


\section{Ranking-based semantics}

An argumentation framework consists of a set of arguments and a set of attacks between them. Arguments represent reasons to believe in statements, doing actions, etc. Attacks express conflicts between pairs of arguments. In what follows, both components are assumed to be abstract entities.

Definition 1 (Argumentation framework) An argumentation framework is an ordered pair $\mathbf{A}=\langle\mathcal{A}, \mathcal{R}\rangle$, where $\mathcal{A}$ is a finite set of arguments and $\mathcal{R}$ a binary relation on $\mathcal{A}$ (i.e., $\mathcal{R} \subseteq \mathcal{A} \times \mathcal{A}$ ). We call $\mathcal{R}$ an attack relation and a $\mathcal{R} b$ means that a attacks $b$.

We turn to the notion of attacker:

Notation Let $\mathbf{A}=\langle\mathcal{A}, \mathcal{R}\rangle$ be an argumentation framework and $a \in \mathcal{A}$. We define that $\operatorname{Arg}(\mathbf{A})=\mathcal{A}$ and $\operatorname{Att}_{\mathbf{A}}(a)=\{b \in \mathcal{A} \mid b \mathcal{R} a\}$. When the context is clear, we write Att (a) for short. The same goes for all notations.

As in classical approaches to argumentation [6], since arguments may be conflicting, it is important to evaluate them and to identify the ones to rely on for inferring conclusions (in case of handling inconsistency in knowledge bases) or making decisions, etc. For that purpose, we propose ranking-based semantics which rank-order the set of arguments from the most acceptable to the weakest one(s). Thus, unlike existing semantics which assign an absolute status (accepted, rejected, undecided) to each argument, the new approach compares pairs of arguments.

Definition 2 (Ranking) $A$ ranking on a set $\mathcal{A}$ is a binary relation $\preceq$ on $\mathcal{A}$ such that: $\preceq$ is total (i.e., $\forall a, b \in \mathcal{A}, a \preceq b$ or $b \preceq a$ ) and transitive (i.e., $\forall a, b, c \in \mathcal{A}$, if $a \preceq b$ and $b \preceq c$, then $a \preceq c$ ). Intuitively, $a \preceq b$ means that $a$ is at least as acceptable $a s b$. So, $b \npreceq$ a means that $a$ is strictly more acceptable than $b$.

We emphasize that, unlike in certain other works, the equal-or-more acceptable argument in an expression of the form $a \preceq b$ is on the left-hand side (i.e., $a$ takes precedence over $b$; the rank of $a$ is above that of $b$; etc.).

Definition 3 (Ranking-based semantics) $A$ ranking-based semantics is a function $\mathbf{S}$ that transforms any argumentation framework $\mathbf{A}=\langle\mathcal{A}, \mathcal{R}\rangle$ into a ranking on $\mathcal{A}$.

A ranking should not be arbitrary, but should obey some postulates. By postulate, we mean any reasonable principle, be it very general or very specific.

\section{Postulates for semantics}

First of all, a ranking on a set of arguments should be defined only on the basis of the attacks between arguments, it should not depend on the identity of the arguments (at least when the data only consist of nodes and arrows). So, our first postulate says that two equivalent argumentation frameworks should give rise to two equivalent rankings. Let us first define the notion of equivalence between two argumentation frameworks. 
Definition 4 (Isomorphism) Let $\mathbf{A}=\langle\mathcal{A}, \mathcal{R}\rangle$ and $\mathbf{A}^{\prime}=\left\langle\mathcal{A}^{\prime}, \mathcal{R}^{\prime}\right\rangle$ be two argumentation frameworks. An isomorphism from $\mathbf{A}$ to $\mathbf{A}^{\prime}$ is a bijective function $f$ from $\mathcal{A}$ to $\mathcal{A}^{\prime}$ such that $\forall a, b \in \mathcal{A}, a \mathcal{R} b$ iff $f(a) \mathcal{R}^{\prime} f(b)$.

We define formally our first postulate and then exemplify it.

Postulate 1 (Abstraction) A ranking-based semantics $\mathbf{S}$ satisfies abstraction $(\mathrm{Ab})$ iff for any two frameworks $\mathbf{A}=\langle\mathcal{A}, \mathcal{R}\rangle$ and $\mathbf{A}^{\prime}=\left\langle\mathcal{A}^{\prime}, \mathcal{R}^{\prime}\right\rangle$, for any isomorphism $f$ from $\mathbf{A}$ to $\mathbf{A}^{\prime}$, we have that $\forall a, b \in \mathcal{A},\langle a, b\rangle \in \mathbf{S}(\mathbf{A})$ iff $\langle f(a), f(b)\rangle \in \mathbf{S}\left(\mathbf{A}^{\prime}\right)$.

Example 1 Consider the two argumentation frameworks depicted in the figure below.
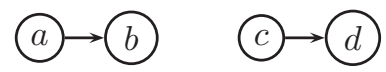

The postulate $(\mathrm{Ab})$ ensures that the ranking relation between $a$ and $b$ is the same as the one between $c$ and $d$.

It is worth pointing out that extension-based semantics (i.e., Dung's semantics) obey in some sense this postulate. For instance, both argumentation frameworks of Example 1 have one preferred extension containing the non-attacked argument ( $a$, resp. $c$ ).

The second postulate states the following: the question whether an argument $a$ is at least as acceptable as an argument $b$ should be independent of any argument $c$ that is neither connected to $a$ nor to $b$, that is, there is no path from $c$ to $a$ or $b$ (ignoring the direction of the edges). Let us first define the independent parts of an argumentation framework.

Definition 5 (Weak connected component) $A$ weak connected component of an argumentation framework $\mathbf{A}$ is a maximal subgraph of $\mathbf{A}$ in which any two vertices are connected to each other by a path (ignoring the direction of the edges). We denote by $\operatorname{Com}(\mathbf{A})$ the set of every argumentation framework $\mathbf{B}$ such that $\mathbf{B}$ is a weak connected component of $\mathbf{A}$ or the graph union of several weak connected components of $\mathbf{A}$.

We turn to our second postulate and to an example.

Postulate 2 (Independence) A ranking-based semantics $\mathbf{S}$ satisfies independence (In) iff for every argumentation framework $\mathbf{A}, \forall \mathbf{B} \in \operatorname{Com}(\mathbf{A}), \forall a, b \in \operatorname{Arg}(\mathbf{B}),\langle a, b\rangle \in$ $\mathbf{S}(\mathbf{A})$ iff $\langle a, b\rangle \in \mathbf{S}(\mathbf{B})$.

Example 1 (Cont) Assume that the two graphs of Example 1 constitute a single argumentation framework. Then, (In) ensures that the ranking relation between $a$ and $b$ (and the one between $c$ and $d$ ) remains the same after the fusion of the two frameworks.

Given our weakening principle (detailed in the introduction), it is natural to consider that a non-attacked argument is more acceptable (and thus ranked higher) than an attacked argument. In other words, there is no full reinstatement for arguments. The third postulate reflects this idea. 
Postulate 3 (Void Precedence) A ranking-based semantics $\mathbf{S}$ satisfies void precedence (VP) iff for every argumentation framework $\mathbf{A}=\langle\mathcal{A}, \mathcal{R}\rangle, \forall a, b \in \mathcal{A}$, if $\operatorname{Att}(a)=\emptyset$ and $\operatorname{Att}(b) \neq \emptyset$, then $\langle b, a\rangle \notin \mathbf{S}(\mathbf{A})$.

Example 1 (Cont) (VP) ensures that $a$ is ranked higher than $b$, and $c$ higher than $d$.

Non-attacked arguments are also favored by extension-based semantics. They belong to any extension under grounded, complete, stable, and preferred semantics. Thus, they are accepted. However, they may have the same status (accepted) as attacked arguments (which are defended). Let us consider the following example.

Example 2 Assume the argumentation framework depicted in the figure below.

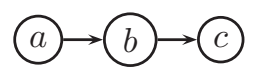

The grounded extension of this framework is $\{a, c\}$. The arguments $a$ and $c$ are both accepted whereas $b$ is rejected. Our approach ranks a higher than $c$ since $c$ is attacked, thus weakened. Thus, it ensures a more refined treatment of arguments.

Since an attack always weakens its target, the next postulate states that having attacked attackers is better than having non-attacked attackers (assuming the number of attackers is the same). In other words, being defended is better than not being defended. First, we formally introduce the notion of defender:

Notation Let $\mathbf{A}=\langle\mathcal{A}, \mathcal{R}\rangle$ be an argumentation framework and $a \in \mathcal{A}$. We denote by $\operatorname{Def}_{\mathbf{A}}(a)$ the set of all defenders of $a$ in $\mathbf{A}$, that is, $\operatorname{Def}_{\mathbf{A}}(a)=\{b \in \mathcal{A} \mid \exists c \in$ $\mathcal{A}, c \mathcal{R} a$ and $b \mathcal{R} c\}$.

Next, we turn to the postulate and to an example.

Postulate 4 (Defense Precedence) A ranking-based semantics $\mathbf{S}$ satisfies defense precedence (DP) ifffor every argumentation framework $\mathbf{A}=\langle\mathcal{A}, \mathcal{R}\rangle, \forall a, b \in \mathcal{A}$, if $|\operatorname{Att}(a)|=$ $|\operatorname{Att}(b)|$, $\operatorname{Def}(a) \neq \emptyset$, and $\operatorname{Def}(b)=\emptyset$, then $\langle b, a\rangle \notin \mathbf{S}(\mathbf{A})$.

Example 3 Consider the argumentation framework depicted in the figure below.
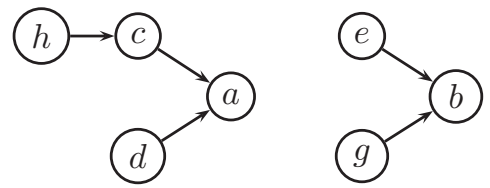

Both arguments $a$ and $b$ have two attackers. The two attackers of $b$ are not attacked, thus they are strong. However, a is defended by $h$, thus the attacker $c$ is weakened. To sum up, a has one strong and one weak attacker, while b has two strong attackers. So, (DP) ensures that a is ranked higher than $b$.

The two next postulates are based on both the weakening and the counting principles: the more the attackers of an argument $a$ are numerous and acceptable, the less $a$ is acceptable. The first postulate, called counter-transitivity, corresponds to a large 
version of this combined principle, the second one, called strict counter-transitivity, corresponds to a strict version.

More precisely, counter-transitivity says that an argument $a$ should be ranked at least as high as an argument $b$, if the attackers of $b$ are at least as numerous and acceptable as those of $a$. Let us first introduce a relation that compares sets of arguments on the basis of a ranking on the arguments.

Definition 6 (Group comparison) Let $\preceq$ be a ranking on a set $\mathcal{A}$ of arguments. For all $A, B \subseteq \mathcal{A},\langle A, B\rangle \in \operatorname{Gr}(\preceq)$ iff there exists an injective function $f$ from $B$ to $A$ such that $\forall a \in B, f(a) \preceq$ a. Intuitively, $\langle A, B\rangle \in \operatorname{Gr}(\preceq)$ iff the elements of the group A are at least as numerous and acceptable as those of $B$.

To put the emphasize on the meaning of $\operatorname{Gr}(\preceq)$, we derive the following fact:

Proposition 1 Let $\preceq$ be a ranking on a set $\mathcal{A}$ of arguments and $A, B \subseteq \mathcal{A}$. If $\langle A, B\rangle \in$ $\operatorname{Gr}(\preceq)$, then:

$-|A| \geq|B|$

- for all $b \in B, \exists a \in A$ such that $a \preceq b$.

We are ready to formally state the postulate based on argument-group comparisons:

Postulate 5 (Counter-Transitivity) A ranking-based semantics $\mathbf{S}$ satisfies the postulate counter-transitivity (CT) iff for every argumentation framework $\mathbf{A}=\langle\mathcal{A}, \mathcal{R}\rangle$, $\forall a, b \in \mathcal{A}$, if $\langle\operatorname{Att}(b), \operatorname{Att}(a)\rangle \in \operatorname{Gr}[\mathbf{S}(\mathbf{A})]$, then $\langle a, b\rangle \in \mathbf{S}(\mathbf{A})$.

Example 3 (Cont) (CT) ensures that $a$ is ranked at least as high as $b$.

Strict counter-transitivity is another mandatory postulate in our approach. Loosely speaking, it says that an argument $a$ should be ranked strictly higher than an argument $b$, if the attackers of $b$ are more numerous or more acceptable than those of $a$.

Definition 7 (Strict group comparison) Let $\preceq$ be a ranking on a set $\mathcal{A}$ of arguments. For all $A, B \subseteq \mathcal{A},\langle A, B\rangle \in \operatorname{Sgr}(\preceq)$ iff there exists an injective function $f$ from $B$ to $A$ such that the two following conditions hold:

- $\forall a \in B, f(a) \preceq a$;

- $|B|<|A|$ or $\exists a \in B, a \npreceq f(a)$.

Intuitively, $\langle A, B\rangle \in \operatorname{Sgr}(\preceq)$ iff the elements of $A$ are strictly better than those of $B$ from a global point of view based on both cardinality and acceptability.

Postulate 6 (Strict Counter-Transitivity) A ranking-based semantics $\mathbf{S}$ satisfies strict counter-transitivity (SCT) iff for every argumentation framework $\mathbf{A}=\langle\mathcal{A}, \mathcal{R}\rangle, \forall a, b \in$ $\mathcal{A}$, if $\langle\operatorname{Att}(b), \operatorname{Att}(a)\rangle \in \operatorname{Sgr}[\mathbf{S}(\mathbf{A})]$, then $\langle b, a\rangle \notin \mathbf{S}(\mathbf{A})$.

Example 3 (Cont) (SCT) ensures that $a$ is strictly more acceptable than $b$.

We turn to situations where the cardinality of the attackers and their quality (i.e., acceptability) are opposed. Here is an example. 
Example 4 Consider the argumentation framework depicted in the figure below.

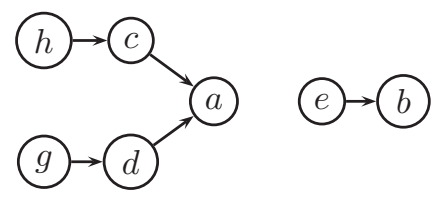

If one non-attacked attacker is sufficient to kill an argument (which is the case in most approaches to argumentation), then the argument $a$ should naturally be ranked higher than $b$. But, in our approach, as explained in the introduction, no number of attacked or non-attacked attackers can kill an argument. They can just weaken it. Consequently, in this example, $a$ is attacked by two weakened arguments, while $b$ is attacked by one strong argument. As usual, we have to make a choice: give precedence to cardinality over quality (i.e. two weakened attackers are worse for the target than one strong attacker), or on the contrary give precedence to quality over cardinality.

In certain applications such as decision-making, both options are reasonable. For example, suppose we have to buy a car and we are considering a red one and a blue one. In addition, the arguments of Example 4 correspond to the following statements:

$b=$ The red car has got 5 stars out of 5 in our favorite car magazine;

$e=$ The magazine does not take into account the fact that the red car is 1000 euros more expensive than the blue one;

$a=$ The blue car has got 5 stars out of 5 in our favorite car magazine;

$c=$ The magazine does not take into account the fact that there is a probability of 0.5 that the blue car engine breaks down before $300000 \mathrm{~km}$. The reparations would cost 2000 euros;

$h=\mathrm{A}$ friend of ours is a mechanic. He would offer us a $10 \%$ discount on engine reparation;

$d=$ The magazine does not take into account the fact that there is a probability of 0.5 that the blue car will be stolen from us before 10 years. The insurance will pay for another blue car, but there is a deductibility provision of 2000 euros;

$g=$ In our neighborhood, the rate of motor vehicle theft is $10 \%$ lower than the average.

In this example, it is intuitive to consider that $b$ is more acceptable than $a$. Indeed, it is obvious that the group $\{c, d\}$ is stronger than the singleton $\{e\}$, despite the fact that the former is slightly weakened by $h$ and $g$. Now, suppose that the argument $e$ is replaced by the following one:

$e=$ The magazine does not take into account the fact that the red car is 4000 euros more expensive than the blue one.

This time it is intuitive to consider that $a$ is more acceptable than $b$.

To summarize, with abstract nodes and arrows as arguments and attacks, the outcome of Example 4 is debatable. We can give precedence to cardinality over quality (i.e. $b$ is more acceptable than $a$ ) or on the contrary give precedence to quality over cardinality (i.e. $a$ is more acceptable than $b$ ). Both options are rational. We turn to two axioms representing these two choices.

First, cardinality precedence says that an argument $a$ should be ranked higher than an argument $b$, if the attackers of $a$ are less numerous than those of $b$. 
Postulate 7 (Cardinality Precedence) A ranking-based semantics $\mathbf{S}$ satisfies cardinality preference (CP) iff for every argumentation framework $\mathbf{A}=\langle\mathcal{A}, \mathcal{R}\rangle, \forall a, b \in \mathcal{A}$, if $|\operatorname{Att}(a)|<|\operatorname{Att}(b)|$, then $\langle b, a\rangle \notin \mathbf{S}(\mathbf{A})$.

Next, quality precedence says that an argument $a$ should be ranked higher than an argument $b$, if at least one attacker of $b$ is ranked higher than any attacker of $a$.

Postulate 8 (Quality Precedence) A ranking-based semantics $\mathbf{S}$ satisfies quality precedence (QP) iff for every argumentation framework $\mathbf{A}=\langle\mathcal{A}, \mathcal{R}\rangle, \forall a, b \in \mathcal{A}$, if there exists $c \in \mathbf{A t t}(b)$ such that $\forall d \in \operatorname{Att}(a),\langle d, c\rangle \notin \mathbf{S}(\mathbf{A})$, then $\langle b, a\rangle \notin \mathbf{S}(\mathbf{A})$.

The last postulate says that, all other things remaining equal, a distributed defense is better than a focused one. This postulate is not at all mandatory. It simply represents a reasonable choice that one can make in very specific situations. More precisely, the idea is to compare two arguments having the same number of attackers and the same number of defenders. In addition, each defender attacks exactly one attacker. The postulate says that, in this case, the best kind of defense is the totally distributed one, i.e. each defender attacks a distinct attacker. In some sense, there is no "overkill".

First, we formally define what is a simple and distributed defense.

Definition 8 (Simple/distributed defense) Let $\mathbf{A}=\langle\mathcal{A}, \mathcal{R}\rangle$ be an argumentation framework and $a \in \mathcal{A}$.

- The defense of a in $\mathbf{A}$ is simple iff every defender of a attacks exactly one attacker of a.

- The defense of a in $\mathbf{A}$ is distributed iff every attacker of a is attacked by at most one argument.

We are ready to define our last postulate:

Postulate 9 (Distributed-Defense Precedence) A ranking-based semantics $\mathbf{S}$ satisfies distributed-defense precedence (DDP) iff for any argumentation framework $\mathbf{A}=\langle\mathcal{A}, \mathcal{R}\rangle$, $\forall a, b \in \mathcal{A}$ such that $|\operatorname{Att}(a)|=|\operatorname{Att}(b)|$ and $|\operatorname{Def}(a)|=|\operatorname{Def}(b)|$, if the defense of $a$ is simple and distributed and the defense of $b$ is simple but not distributed, then $\langle b, a\rangle \notin \mathbf{S}(\mathbf{A})$.

Let us illustrate these concepts on the following example.

Example 5 Consider the argumentation framework depicted in the figure below.
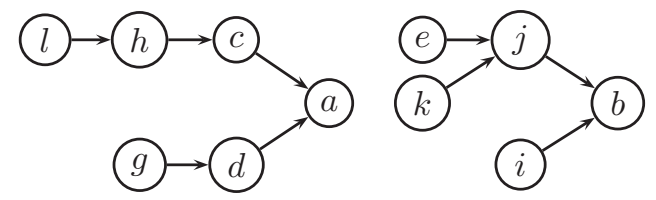

The two arguments $a$ and $b$ have the same number of defenders: $\operatorname{Def}(a)=\{h, g\}$ and $\operatorname{Def}(b)=\{e, k\}$. However, the defense of $a$ is simple and distributed while the defense of $b$ is simple but not distributed. The postulate (DDP) ensures that a is more acceptable than $b$, despite the fact that the defenders of a are weaker than those of $b$. 


\section{Relationships between postulates}

So far we have proposed a set of postulates that are suitable for defining a rankingbased semantics in argumentation theory. In the present section, we briefly study their dependencies, as well as their compatibilities (i.e., whether they can be satisfied together by a semantics). We start by showing that the postulates (CT), (SCT), (VP) and (DP) are not independent.

Proposition 2 Let $\mathbf{S}$ be a ranking-based semantics:

- if $\mathbf{S}$ satisfies (SCT), then it satisfies (VP);

- if $\mathbf{S}$ satisfies both (CT) and (SCT), then it satisfies (DP).

Let us now check the compatibility of the postulates. Unsurprisingly, (CP) and (QP) cannot be satisfied together. Example 4 already illustrates this issue. Indeed, (QP) prefers $a$ to $b$, while (CP) prefers the converse.

Proposition 3 No ranking-based semantics can satisfy both (CP) and (QP).

In the next section, we construct a ranking-based semantics showing the following compatibility result:

Proposition 4 The postulates $(\mathrm{Ab}),(\mathrm{In}),(\mathrm{CT}),(\mathrm{SCT}),(\mathrm{CP})$, and (DDP) are compatible.

\section{Discussion-based and Burden-based semantics}

This section introduces two semantics satisfying most of our postulates, namely those that are compatible with $(\mathrm{CP})$.

The first semantics, called discussion-based semantics, is centered on a notion of linear discussion similar to 'argumentation line' in [8]. A linear discussion is a sequence of arguments such that each argument attacks the argument preceding it in the sequence.

Definition 9 (Linear discussions) Let $\mathbf{A}=\langle\mathcal{A}, \mathcal{R}\rangle$ be an argumentation framework and $a \in \mathcal{A}$. $A$ linear discussion for $a$ in $\mathbf{A}$ is a sequence $s=\left\langle a_{1}, \ldots, a_{n}\right\rangle$ of elements of $\mathcal{A}$ (where $n$ is a positive integer) such that $a_{1}=a$ and $\forall i \in\{2,3, \ldots, n\} a_{i} \mathcal{R} a_{i-1}$. The length of $s$ is $n$. We say that: $s$ is won iff $n$ is odd; $s$ is lost iff $n$ is even.

Let us illustrate this notion on an example.

Example 5 (Cont) Two won linear discussions for the argument $a$ are e.g., $s_{1}=\langle a\rangle$ and $s_{2}=\langle a, d, g\rangle$ and one lost linear discussion is, for instance, $s_{3}=\langle a, c, h, l\rangle$. Similarly, three won linear discussions for the argument $b$ are $s_{1}^{\prime}=\langle b\rangle, s_{2}^{\prime}=\langle b, j, e\rangle$ and $s_{3}^{\prime}=\langle b, j, k\rangle$ and one lost discussion is $s_{4}^{\prime}=\langle b, i\rangle$.

The basic idea behind the semantics is the following: for every argument $a$, for every positive integer $i$, we count the number of linear discussions for $a$ of length $i$. We positively count the lost discussions and negatively count the won discussions. So, in any case, the smaller the number calculated, the better the situation for $a$. 
Definition 10 (Discussion count) Let $\mathbf{A}=\langle\mathcal{A}, \mathcal{R}\rangle$ be an argumentation framework, $a \in \mathcal{A}$, and $i$ a positive integer. We define that:

$$
\operatorname{Dis}_{\mathbf{A} i}(a)=\left\{\begin{array}{l}
-N \text { if } i \text { is odd } \\
N \quad \text { if } i \text { is even }
\end{array}\right.
$$

where $N$ is the number of linear discussions for a in $\mathbf{A}$ of length $i$.

Example 5 (Cont) The following table provides the discussion counts $\operatorname{Dis}_{\mathrm{A} i}$ of the two arguments $a$ and $b$.

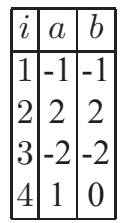

Our strategy is to lexicographically rank the arguments on the basis of their won and lost linear discussions.

Definition 11 (Discussion-based semantics) The ranking-based semantics Dbs transforms any argumentation framework $\mathbf{A}=\langle\mathcal{A}, \mathcal{R}\rangle$ into the ranking $\operatorname{Dbs}(\mathbf{A})$ on $\mathcal{A}$ such that $\forall a, b \in \mathcal{A},\langle a, b\rangle \in \operatorname{Dbs}(\mathbf{A})$ iff one of the two following cases holds:

- $\forall i \in\{1,2, \ldots\}, \operatorname{Dis}_{i}(a)=\operatorname{Dis}_{i}(b)$;

- $\exists i \in\{1,2, \ldots\}, \operatorname{Dis}_{i}(a)<\operatorname{Dis}_{i}(b)$ and $\forall j \in\{1,2, \ldots, i-1\}$, $\operatorname{Dis}_{j}(a)=\operatorname{Dis}_{j}(b)$.

Example 5 (Cont) For every $i \in\{1,2,3\}$, $\operatorname{Dis}_{i}(a)=\operatorname{Dis}_{i}(b)$. However, $\operatorname{Dis}_{4}(a)>$ $\operatorname{Dis}_{4}(b)$. Thus, $\langle a, b\rangle \notin \operatorname{Dbs}(\mathbf{A})$, i.e., $b$ is strictly more acceptable than $a$.

At first sight, the infinite character of the set $\{1,2, \ldots\}$ of all positive integers may look like an issue from a computational point of view. Indeed, $\operatorname{Dis}_{i}(a)$ may never stop evolving. This is due to the possible presence of cycles in the argumentation framework. But, if $\operatorname{Dis}_{i}(a)$ never stops evolving, it evolves cyclically. So, we strongly conjecture that there exists a threshold $t$ such that if $\forall i \leq t$, $\operatorname{Dis}_{i}(a)=\operatorname{Dis}_{i}(b)$, then $\forall i>t$, $\operatorname{Dis}_{i}(a)=\operatorname{Dis}_{i}(b)$. Such an equality-ensuring threshold would be dependent on the length of the longest elementary cycle in the argumentation framework. This threshold would be useful to write a program implementing our discussion-based semantics.

Note also that the computation can simply be done up to a fixed step $t$. The greater $t$, the closer the ranking obtained to the actual discussion-based ranking.

Next, the postulates represent theoretical validations for our semantics:

Theorem 1 Dbs satisfies (Ab), (In), (CT), (SCT), and (CP).

From Proposition 2, it is immediate that Dbs satisfies additional postulates:

Corollary 1. Dbs satisfies (VP) and (DP).

Theorem 2 Dbs does not satisfy (DDP). 
Next, we show that Dbs treats odd and even length cycles in a similar way:

Proposition 5 Let $\mathbf{A}=\langle\mathcal{A}, \mathcal{R}\rangle$ be an argumentation framework. Suppose that $\mathbf{A}$ takes the form of a unique cycle, i.e. there exists an enumeration $\left\langle a_{1}, \ldots, a_{n}\right\rangle$ of $\mathcal{A}$ (without repetition and where $n$ is a positive integer) such that $\forall i \in\{1,2, \ldots, n-1\}, \operatorname{Att}\left(a_{i}\right)=$ $\left\{a_{i+1}\right\}$, and $\operatorname{Att}\left(a_{n}\right)=\left\{a_{1}\right\}$. Then, $\forall a, b \in \mathcal{A},\langle a, b\rangle \in \operatorname{Dbs}(\mathbf{A})$.

The second semantics, called burden-based semantics, satisfies (DDP). It follows a multiple steps process. At each step, it assigns a burden number to every argument. In the initial step, this number is 1 for all arguments. Then, in each step, all the burden numbers are simultaneously recomputed on the basis of the number of attackers and their burden numbers in the previous step. More precisely, for every argument $a$, its burden number is set back to 1 , then, for every argument $b$ attacking $a$, the burden number of $a$ is increased by a quantity inversely proportional to the burden number of $b$ in the previous step. More formally:

Definition 12 (Burden numbers) Let $\mathbf{A}=\langle\mathcal{A}, \mathcal{R}\rangle$ be an argumentation framework, $i \in\{0,1, \ldots\}$, and $a \in \mathcal{A}$. We denote by $\operatorname{Bur}_{\mathbf{A} i}(a)$ the burden number of $a$ in the $i^{\text {th }}$ step, i.e.:

$$
\operatorname{Bur}_{i}(a)= \begin{cases}1 & \text { if } i=0 \\ 1+\Sigma_{b \in \operatorname{Att}(a)} 1 / \operatorname{Bur}_{i-1}(b) & \text { otherwise. }\end{cases}
$$

By convention, if $\operatorname{Att}(a)=\emptyset$, then $\Sigma_{b \in \operatorname{Att}(a)} 1 / \operatorname{Bur}_{i-1}(b)=0$.

Let us illustrate this function on the following example.

Example 2 (Cont) The burden numbers of each argument are summarized in the table below. Note that these numbers will not change beyond step 2 .

\begin{tabular}{|c|c|c|c|}
\hline Step $i$ & $a$ & $b$ & $c$ \\
\hline 0 & 1 & 1 & 1 \\
1 & 1 & 2 & 2 \\
2 & 1 & 2 & 1.5 \\
$\vdots$ & $\vdots$ & $\vdots$ & $\vdots$ \\
\hline
\end{tabular}

We lexicographically compare two arguments on the basis of their burden numbers.

Definition 13 (Burden-based semantics) The ranking-based semantics Bbs transforms any argumentation framework $\mathbf{A}=\langle\mathcal{A}, \mathcal{R}\rangle$ into the ranking $\operatorname{Bbs}(\mathbf{A})$ on $\mathcal{A}$ such that $\forall a, b \in \mathcal{A},\langle a, b\rangle \in \operatorname{Bbs}(\mathbf{A})$ iff one of the two following cases holds:

- $\forall i \in\{0,1, \ldots\}, \operatorname{Bur}_{i}(a)=\operatorname{Bur}_{i}(b)$;

- $\exists i \in\{0,1, \ldots\}, \operatorname{Bur}_{i}(a)<\operatorname{Bur}_{i}(b)$ and $\forall j \in\{0,1, \ldots, i-1\}, \operatorname{Bur}_{j}(a)=\operatorname{Bur}_{j}(b)$.

As for the discussion-based semantics, an equality-ensuring threshold probably exists for the burden-based semantics. Such a threshold would make possible an exact computation, despite the fact that $\{0,1, \ldots\}$ is infinite.

Note that both semantics (Dbs and Bbs) do not take into account possible dependencies between an argument and one of its attackers, nor the dependencies between 
two attackers. Actually, Dbs and Bbs rank the arguments only on the basis of the structure obtained by "unrolling" the cycles. For example, our semantics do not distinguish between a loop (e.g. $a \mathcal{R} a$ ) and a cycle (e.g. $a \mathcal{R} b, b \mathcal{R} a$ ). The notion of dependence is hard to capture and beyond the scope of this paper. Our goal in the present paper is essentially to introduce a new kind of semantics, basic postulates for it, and instances satisfying those postulates.

We turn to the postulate-based analysis of Bbs:

Theorem 3 Bbs satisfies (Ab), ( In), (CT), (SCT), (CP), and (DDP).

From Proposition 2, it satisfies more postulates:

Corollary 2. Bbs satisfies (VP) and (DP).

Let us see on examples how the semantics works.

Example 2 (Cont) According to Bbs, the argument $a$ is strictly more acceptable than $c$ which is itself strictly more acceptable than $b$.

Note that Bbs returns a more refined result than Dung's semantics. Indeed, the set $\{a, c\}$ is a (preferred, grounded, stable) extension according to [6]. Our approach refines the result by ranking $a$ higher than $c$ since it is not attacked. This does not mean that Bbs semantics coincides with Dung's ones. The following example shows that the two approaches may return different results since they are grounded on different principles.

Example 4 (Cont) The argumentation framework has a unique extension $\{h, g, a, e\}$ which is grounded, preferred and stable. Thus, the argument $b$ is rejected. Let us now apply the Bbs semantics on the same framework. The table below provides the burden numbers of the arguments.

\begin{tabular}{|c|c|c|c|c|c|c|c|}
\hline Step $i$ & $h$ & $g$ & $c$ & $d$ & $a$ & $e$ & $b$ \\
\hline 0 & 1 & 1 & 1 & 1 & 1 & 1 & 1 \\
1 & 1 & 1 & 2 & 2 & 3 & 1 & 2 \\
2 & 1 & 1 & 2 & 2 & 2 & 1 & 2 \\
$\vdots$ & $\vdots$ & $\vdots$ & $\vdots$ & $\vdots$ & $\vdots$ & $\vdots$ & $\vdots$ \\
\hline
\end{tabular}

Bbs provides the following ranking: $h, g, e \preceq c, d, b \preceq a$. Thus, $b$ is more acceptable than $a$. The reason is that $b$ has less attackers and $\mathrm{Bbs}$ give precedence to the cardinality of the attackers over their quality.

Example 5 (Cont) According to Bbs, $a$ is strictly more acceptable than $b$.

Note that in this example, the semantics Dbs returns the converse. This shows that the two semantics may return very different results. This difference comes from the postulate DDP which is satisfied by Bbs but violated by Dbs.

As with Dbs, we show next that the Bbs semantics treats odd and even length cycles in a similar way. 
Proposition 6 Let $\mathbf{A}=\langle\mathcal{A}, \mathcal{R}\rangle$ be an argumentation framework. Suppose that $\mathbf{A}$ takes the form of a unique cycle, i.e. there exists an enumeration $\left\langle a_{1}, \ldots, a_{n}\right\rangle$ of $\mathcal{A}$ (without repetition and where $n$ is a positive integer) such that $\forall i \in\{1,2, \ldots, n-1\}, \operatorname{Att}\left(a_{i}\right)=$ $\left\{a_{i+1}\right\}$, and $\operatorname{Att}\left(a_{n}\right)=\left\{a_{1}\right\}$. Then, $\forall a, b \in \mathcal{A},\langle a, b\rangle \in \operatorname{Bbs}(\mathbf{A})$.

\section{Related work}

There are three works in the literature which are somehow related to our contribution. The first attempts were done in $[1,2]$ where the authors identified different principles and compared existing semantics wrt them. The principles are tailored for extensionbased semantics, and do not apply for ranking-based ones.

The work in [4] is closer to ours. The authors defined a notion of gradual acceptability. The idea is to assign a numerical value to each argument on the basis of its attackers. The properties of the valuation function are unclear. Our approach defines, through a set of formal postulates, the desirable properties of our semantics.

In [7], Dung's abstract framework was extended by considering weighted attacks. The basic idea is to remove some attacks up to a certain degree representing the tolerated incoherence, and then apply existing semantics to the new graph(s) by ignoring completely the weights. This leads to extensions which are not conflict-free in the sense of the attack relation. Consider the following weighted framework. If one tolerates incoherence up to degree $1(\beta=1)$, then the attack from $a$ to $b$ is ignored. Consequently, $\emptyset$ and $\{a, b\}$ are two $\beta$-grounded extensions.

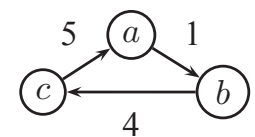

This approach is different from ours for several reasons.

First, it does not obey the four graded considerations at the basis of our postulates and semantics (i.e., weakening, counting, relativity, and graduality), it rather obeys the four traditional non-graded considerations described in the introduction (i.e., killing, existence, absoluteness, and flatness). Indeed, weights are only used for deciding which attacks can be ignored when computing the extensions.

The second main difference stems from the fact that weights of attacks are inputs of the argumentation system of [7]. In our approach, degrees are located in the output, i.e. we compute the relative degree of acceptability of each argument. Note that the more an argument is acceptable, the more the attacks emanating from it are important. However, this does not mean that weights of attacks are generated. In our approach, the three arguments $a, b$ and $c$ are equivalent with regard to Bbs and Dbs. Finally, our semantics can be extended to deal with weighted attacks as input.

\section{Conclusion}

The paper develops an axiomatic approach for defining semantics for argumentation frameworks. It proposes postulates (each of which represents a criterion) that a semantics may satisfy. The approach offers thus a theoretical framework for comparing semantics. It is worth emphasizing that only some of the postulates (e.g. abstraction) are 
satisfied by Dung's semantics (when the arguments are ranked on the basis of their status, i.e. accepted arguments are ranked above undecided ones, which are ranked above rejected ones). The other postulates are based on graded considerations which may be natural in applications like decision-making.

Another novelty of our approach is that it computes the acceptability of arguments without passing through multiple points of view. Its basic idea is to compute a complete ranking on the set of arguments. The paper proposes two novel semantics that satisfy the postulates but that do not necessarily return the same results. An important future work is to find sufficiently many postulates to characterize our semantics.

\section{References}

1. L. Amgoud and S. Vesic. A new approach for preference-based argumentation frameworks. Annals of Mathematics and Artificial Intelligence, 63(2):149-183, 2011.

2. P. Baroni and M. Giacomin. On principle-based evaluation of extension-based argumentation semantics. Artificial Intelligence, 171(10-15):675-700, 2007.

3. P. Baroni, M. Giacomin, and G. Guida. Scc-recursiveness: a general schema for argumentation semantics. Artificial Intelligence Journal, 168:162-210, 2005.

4. C. Cayrol and M.-C. Lagasquie-Schiex. Graduality in Argumentation. Journal of Artificial Intelligence Research (JAIR), 23:245-297, 2005.

5. P. Dung, P. Mancarella, and F. Toni. Computing ideal skeptical argumentation. Artificial Intelligence Journal, 171:642-674, 2007.

6. P. M. Dung. On the Acceptability of Arguments and its Fundamental Role in Non-Monotonic Reasoning, Logic Programming and n-Person Games. AIJ, 77:321-357, 1995.

7. P. E. Dunne, A. Hunter, P. McBurney, S. Parsons, and M. Wooldridge. Weighted argument systems: Basic definitions, algorithms, and complexity results. Artificial Intelligence, 175(2):457-486, 2011.

8. A. García and G. Simari. Defeasible logic programming: an argumentative approach. Theory and Practice of Logic Programming, 4(1-2):95-138, 2004. 